\title{
Systems with selective overflow and change of bandwidth
}

\author{
Iversen, Villy Bæk
}

Published in:

First IEEE International Conference on Communications in China: Wireless Networking and Applications (WNA)

Link to article, DOI:

10.1109/ICCChina.2012.6356973

Publication date:

2012

Link back to DTU Orbit

\section{Citation (APA):}

Iversen, V. B. (2012). Systems with selective overflow and change of bandwidth. In First IEEE International Conference on Communications in China: Wireless Networking and Applications (WNA) (pp. 694-697). IEEE. https://doi.org/10.1109/ICCChina.2012.6356973

\section{General rights}

Copyright and moral rights for the publications made accessible in the public portal are retained by the authors and/or other copyright owners and it is a condition of accessing publications that users recognise and abide by the legal requirements associated with these rights.

- Users may download and print one copy of any publication from the public portal for the purpose of private study or research.

- You may not further distribute the material or use it for any profit-making activity or commercial gain

- You may freely distribute the URL identifying the publication in the public portal

If you believe that this document breaches copyright please contact us providing details, and we will remove access to the work immediately and investigate your claim. 


\title{
Systems with selective overflow and change of bandwidth
}

\author{
Villy B. Iversen \\ Department of Photonics Engineering, Technical University of Denmark \\ DK-2800 Kongens Lyngby, Denmark \\ Email: vbiv@fotonik.dtu.dk
}

\begin{abstract}
We consider a loss system with $n$ channels and a finite or infinite overflow group, which is offered $N$ different services, all having Poisson arrival processes. All calls have same bandwidth demand and mean service time, but the mean service time may be different on the primary group and the overflow group, corresponding to data traffic with different bandwidth allocation on primary (micro-cell $=$ femto-cell) and overflow group (macro-cell $=$ LTE-cell). Then using a result of Wallström we can calculate the Binomial moments of the total overflow traffic. Given a certain number of busy channels on the overflow group, we show by balance equations that the number of calls of each service will be Multinomial distributed with probabilities proportional with the arrival rates. Using a recent result of Newcomer \& al, we then find moments (done up to fourth order) of individual overflow streams or any combinations of overflow streams. Thus we can find the correlation between services and for example the moments of some traffic streams which may overflow to one system, whereas other traffic streams may be blocked or overflow to another system.

Keywords: partial overflow traffic, changing mean holding times, changing bandwidth, femto-cells, overlay-cell
\end{abstract}

\section{INTRODUCTION}

This paper presents a generalization of more classical overflow problems. We derive moments and correlations of combined overflow traffic streams from a trunk group. Furthermore, we allows the bandwidth (and thus the mean holding time) to change from primary group to overflow group. This appears for example for file-transfer in hierarchical cellular systems where the allocated bandwidth may change from femto-cell to overlaid LTE-cell.

The combination of correlated traffic streams was first dealt with by Descloux [4] and Neal [6], resulting in the Extended Equivalent Random Traffic method. The paper by Zhao \& Gambe [11] dealt with only two traffic streams. Recently Schneps-Schneppe \& Sedols [9] have made further contributions to this field. Only two first moments are considered and the exact solutions are very complex. The change of mean holding time (equivalently change of bandwidth) was first consider by Wallström [10] and has later been dealt with by Schehrer [8] and Bakmaz [1] [2]. They assume Poisson arrival processes. In Lindkvist [5] the results are generalized to Interrupted Poisson arrival processes. In general, the authors derive very complex solutions based on generating functions.

In this paper we deal with both correlated traffic streams and change of bandwidth in the same model. In Section II we recall Wallström's solution from 1973 which yields the Binomial moments of the overflow traffic in a system with Poisson arrival process with rate $\lambda$ and different mean values of the exponential service times on the primary group $\left(1 / \mu_{p}\right)$ and overflow group group $\left(1 / \mu_{o}\right)$. We also summarize relationships between ordinary moments, Binomial moments, and factorial moments, which all are widely used in overflow theory. In Section III we assume that the Poisson arrival process with arrival rate $\lambda$ is composed of $N$ arrival streams with arrival rates $\lambda_{j}, j=1, N, \sum \lambda_{j}=\lambda$. All streams have the same mean holding time $1 / \mu_{p}$ on the primary group and same mean holding time $1 / \mu_{o}$ on the overflow group. We then assume that the detailed state probabilities on the overflow group for at fixed global state is given by the Multinomial distribution. This is proved to be correct by showing that the node balance equations are fulfilled. In Section IV we finally exploit recent results on Multinomial distributions by Newcomer \& al [7] to derive moments up to the order four for a system with four traffic streams for all combinations of these four streams. Thus we for example are able to find the moments of overflow traffic where two traffic streams are allowed to overflow whereas the other two are blocked or routed to a third system.

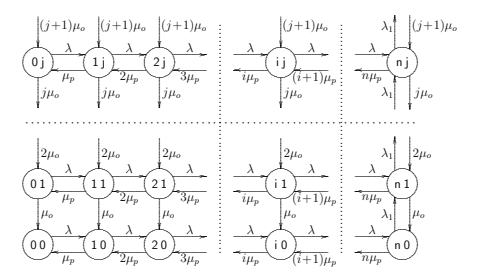

Fig. 1. State transition diagram for a generalized Kosten's system, which has a primary group with $n$ channels and an unlimited overflow group. The states are denoted by $[x, y]$, where $x$ is the number of busy channels in the primary group, and $y$ is the number of busy channels in the overflow group. The service rate is $\mu_{p}$ on the primary group and $\mu_{o}$ on the overflow group.

\section{STATE PROBABILITIES AND MOMENTS OF OVERFLOW} TRAFFIC

Wallström (1973 [10] consider an overflow system with a primary group of $n$ channels and an overflow group with $\ell$ channels. The holding times in the primary group are exponentially distributed with mean value $1 / \mu_{p}$ and the holding time in the overflow group is exponentially distributed with mean value $1 / \mu_{o}$. He derive a recursion formula for the $i$ 'th 
Binomial moment $\beta_{i}$ of the traffic offered to the overflow group:

$$
\begin{aligned}
\beta_{i} & =\frac{\left(\frac{\lambda}{\mu_{o}}\right)^{i}}{i !} \cdot \prod_{j=0}^{i-1} \kappa_{j}(n), \quad \text { where } \\
\kappa_{j}(x) & =\frac{\frac{\lambda}{\mu_{o}} \cdot \kappa_{j}(x-1)+j}{\frac{\mu_{p}}{\mu_{o}} \cdot x+\frac{\lambda}{\mu_{o}} \cdot \kappa_{j}(x-1)+j}, \quad \kappa_{j}(0)=1
\end{aligned}
$$

The blocking probability is obtained from

$$
\begin{aligned}
P(n, \ell) & =\frac{\kappa_{\ell}(n)}{b_{\ell}}, \text { where } \\
b_{0} & =1 \\
b_{i} & =\frac{1}{\kappa_{\ell-i}(n)}\left(\frac{\mu_{o}}{\lambda}(\ell-i+1) \cdot b_{i-1}+\left(\begin{array}{l}
\ell \\
i
\end{array}\right) \cdot \kappa_{\ell}(n)\right)
\end{aligned}
$$

In overflow theory we usually for convenience use Binomial moments $\beta_{i}$. Later in this paper we also consider factorial moments $\varphi_{i}$. For discrete non-negative distributions $p(x)$ we have the following relationships between ordinary (raw) moments $m_{i}$ and the above types of moments:

$$
\begin{aligned}
m_{i} & =\sum_{x=0}^{\infty} x^{i} p(x) \\
\varphi_{i} & =\sum_{x=0}^{\infty} \frac{x !}{(x-i) !} p(x) \\
\beta_{i} & =\sum_{x=0}^{\infty}\left(\begin{array}{l}
x \\
i
\end{array}\right) p(x)
\end{aligned}
$$

The $i$ 'th factorial moment $\varphi_{i}$ is obtained from the Binomial moment by:

$$
\varphi_{i}=i ! \cdot \beta_{i}
$$

Expressed by the raw moments $m_{i}$ we also have:

$$
\begin{aligned}
& \varphi_{1}=m_{1} \\
& \varphi_{2}=m_{2}-m_{1} \\
& \varphi_{3}=m_{3}-3 m_{2}+2 m_{1} \\
& \varphi_{4}=m_{4}-6 m_{3}+11 m_{2}-6 m_{1}
\end{aligned}
$$

The reverse way we have:

$$
\begin{aligned}
& m_{1}=\varphi_{1} \\
& m_{2}=\varphi_{2}-\varphi_{1} \\
& m_{3}=\varphi_{3}+3 \varphi_{2}-5 \varphi_{1} \\
& m_{4}=\varphi_{4}+6 \varphi_{3}+7 \varphi_{2}-47 \varphi_{1}
\end{aligned}
$$

\section{Multinomial State PROBABilities}

In this section we show, that given $x$ channels are busy on the primary group, all with mean service rate $\mu_{p}$, and $y$ channels are busy on the overflow group, all with mean service rate $\mu_{o}$, then the number of calls of each service on the the overflow group will be Multinomial-distributed. This is simply shown by considering node flow balance equations of the state transition diagram shown in Fig. 1.

\section{A. Flow balance equations}

Let the global state probabilities be given by $P(x, y)$ where $x$ is the number of busy channels in the primary group and $y$ is the number of channels on the overflow group. Let us split the state probabilities on the overflow group into detailed state probabilities $\{y\}=\left\{y_{1}, y_{2}, \ldots, y_{N}\right\}$. Then we can show that:

$$
\begin{aligned}
P\left(x, y_{1}, y_{2}, \ldots, y_{N} \mid y\right)= & P(x, y) \cdot\left(\begin{array}{c}
y \\
y_{1}, y_{2}, \ldots, y_{N}
\end{array}\right) \\
& \cdot p_{1}^{y_{1}} \cdot p_{2}^{y_{2}} \cdot \ldots \cdot p_{N}^{y_{N}}, \quad(15)
\end{aligned}
$$

where

$$
\begin{aligned}
p_{i} & =\frac{\lambda_{i}}{\lambda}, \\
y & =\sum_{i=1}^{N} y_{i} .
\end{aligned}
$$

Proof for states $P(x, y), x<n$ :

For states $\left\{x, y_{1}, y_{2}, \ldots, y_{N} \mid y\right\}$ we have the following flow balance equations for $0 \leq x<n$ :

$$
0=-F_{\text {out }}(x, y)+F_{1, \text { in }}(x, y)+F_{2, \text { in }}(x, y)+F_{3, \text { in }}(x, y)
$$

where the following state transitions are feasible:

- Flow out due to arrival or departure:

$F_{\text {out }}(x, y)=\left(\lambda+x \cdot \mu_{p}+y \cdot \mu_{o}\right) \cdot P\left(x, y_{1}, y_{2}, \ldots, y_{N} \mid y\right)$

- Flow in due to arrival (will always be to primary group because $x<n$ ):

$$
F_{1, i n}(x, y)=\lambda \cdot P\left(x-1, y_{1}, y_{2}, \ldots, y_{N} \mid y\right)
$$

- Flow in due to departure from primary group:

$$
F_{2, \text { in }}(x, y)=(x+1) \cdot \mu_{p} \cdot P\left(x+1, y_{1}, y_{2}, \ldots, y_{N} \mid y\right)
$$

- Flow in due to departure from overflow group:

$$
\begin{aligned}
& F_{3, \text { in }}(x, y)=\sum_{i=1}^{N}\left(y_{i}+1\right) \cdot \mu_{o} \\
& \quad \cdot P\left(x, y_{1}, y_{2}, \ldots, y_{i-1}, y_{i}+1, y_{i+1}, \ldots, y_{N} \mid y+1\right)
\end{aligned}
$$

By inserting (15) into (16) we show that the balance equations are fulfilled. Inserting (15) into $F_{3, \text { in }}(x, y)$ yields:

$$
\begin{aligned}
F_{3, i n}(n, y)= & \sum_{i=1}^{N}\left(y_{i}+1\right) \cdot \mu_{o} \cdot P(x, y+1) \\
& \cdot\left(\begin{array}{c}
y_{1}, y_{2}, \ldots, y_{i-1}, y_{i}+1, y_{i+1}, \ldots, y_{N}
\end{array}\right) \\
& \cdot p_{1}^{y_{1}} \cdot p_{2}^{y_{2}} \cdots p_{i-1}^{y_{i-1}} \cdot y_{i}^{y_{i}+1} \cdot p_{i+1}^{y_{i+1}} \cdots p_{N}^{y_{N}} \\
F_{3, i n}(n, y)= & (y+1) \cdot \mu_{o} \cdot P(x, y+1) \\
& \cdot \sum_{i=1}^{N}\left\{\left(\begin{array}{c}
y_{1}, y_{2}, \ldots, y_{i}, \ldots, y_{N}
\end{array}\right)\right. \\
& \left.\cdot p_{1}^{y_{1}} \cdot p_{2}^{y_{2}} \cdots p_{i-1}^{y_{i-1}} \cdot y_{i}^{y_{i}+1} \cdot p_{i+1}^{y_{i+1}} \cdots p_{N}^{y_{N}}\right\} \\
= & (y+1) \cdot \mu_{o} \cdot P(x, y+1) \\
& \cdot\left(\begin{array}{c}
y_{1}, y_{2}, \ldots, y_{i}, \ldots, y_{N}
\end{array}\right) \\
& \cdot p_{1}^{y_{1}} \cdot p_{2}^{y_{2}} \ldots y_{i}^{y_{i}} \cdots p_{N}^{y_{N}}
\end{aligned}
$$


as we have $\sum_{i=1}^{N} p_{i}=1$. Inserting this into the flow balance equation (16) together with (15) we get after reducing all terms with same factors:

$$
\begin{aligned}
0= & -\left(\lambda+x \cdot y+y \cdot \mu_{o}\right) \cdot P(x, y) \\
& +\lambda \cdot P(x-1, y) \\
& +(x+1) \cdot \mu_{p} \cdot P(x+1, y) \\
& +(y+1) \cdot \mu_{o} \cdot P(x, y+1)
\end{aligned}
$$

This is the global balance equations for states $P(x, y), x<n$, which thus are fulfilled. From the theory of Markov processes we know that there is one and only one solution. Thus the state probabilities in this case are given by (15).

Proof for states $\boldsymbol{P}(\boldsymbol{n}, \boldsymbol{y})(x=n)$

The node flow balance equation is:

$$
0=-F_{\text {out }}(n, y)+F_{1, i n}(n, y)+F_{2, i n}(n, y)+F_{3, i n}(n, y)
$$

where the following state transitions are feasible:

- Flow out due to arrival or departure: $F_{\text {out }}(n, y)=\left(\lambda+n \cdot \mu_{p}+y \cdot \mu_{o}\right) \cdot P\left(n, y_{1}, y_{2}, \ldots, y_{N} \mid y\right)$

- Flow in due to arrival to primary group: $F_{1, i n}(n, y)=\lambda \cdot P\left(n-1, y_{1}, y_{2}, \ldots, y_{N} \mid y\right)$

- Flow in due to arrival to overflow group: $F_{2, i n}(n, y)=\sum_{i=1}^{N} \lambda_{i}$

$$
\text { . } P\left(n, y_{1}, y_{2}, \ldots, y_{i-1}, y_{i}-1, y_{i+1}, \ldots, y_{N} \mid y-1\right)
$$

- Flow in due to departure (will always be from overflow group):

$$
\begin{aligned}
& F_{3, i n}(n, y)=\sum_{i=1}^{N}\left(y_{i}+1\right) \cdot \mu_{o} \\
& \quad \cdot P\left(n, y_{1}, y_{2}, \ldots, y_{i-1}, y_{i}+1, y_{i+1}, \ldots, y_{N} \mid y+1\right)
\end{aligned}
$$

The expression (18) is also valid for $F_{3, i n}(n, y+1)$, i.e. for $x=n$. The expression (19) includes a term $F_{2, i n}(n, y)$ which includes state $y-1$. Inserting (15) into $F_{2, i n}(n, y)$ yields:

$$
\begin{aligned}
F_{2, i n}(n, y)= & \sum_{i=1}^{N} \lambda_{i} \cdot P(n, y-1) \\
& \cdot\left(\begin{array}{c}
y-1 \\
y_{1}, y_{2}, \ldots, y_{i-1}, y_{i}-1, y_{i+1}, \ldots, y_{N}
\end{array}\right) \\
& \cdot p_{1}^{y_{1}} \cdot p_{2}^{y_{2}} \cdots p_{i-1}^{y_{i-1}} \cdot y_{i}^{y_{i}-1} \cdot p_{i+1}^{y_{i+1}} \cdots p_{N}^{y_{N}} \\
= & \sum_{i=1}^{N} \lambda_{i} \cdot P(n, y-1) \cdot \frac{y_{i}}{y} \\
& \cdot\left(\begin{array}{c}
y_{1}, y_{2}, \ldots, y_{i}, \ldots, y_{N}
\end{array}\right) \\
& \cdot \frac{1}{p_{i}} p_{1}^{y_{1}} \cdot p_{2}^{y_{2}} \cdots y_{i}^{y_{i}} \ldots p_{N}^{y_{N}} \\
= & \lambda \cdot P(n, y-1) \cdot\left(\begin{array}{c}
y_{1}, y_{2}, \ldots, y_{i}, \ldots, y_{N}
\end{array}\right) \\
& \cdot p_{1}^{y_{1}} \cdot p_{2}^{y_{2}} \ldots y_{i}^{y_{i}} \cdots p_{N}^{y_{N}},
\end{aligned}
$$

because we have $\sum_{i=1}^{N} \lambda_{i} \cdot \frac{y_{i}}{y} \cdot \frac{1}{p_{i}}=\lambda$.

Inserting the obtained expressions for $F_{2, i n}(n, y)$ and $F_{3, \text { in }}(n, y+1)$ into (19) and using (15), we get after reducing the factors common to all terms:

$$
\begin{aligned}
0= & -\left(\lambda+n \cdot y+y \cdot \mu_{o}\right) \cdot P(n, y) \\
& +\lambda \cdot P(n-1, y) \\
& +\lambda \cdot P(n, y-1) \\
& +(y+1) \cdot \mu_{o} \cdot P(x, y+1)
\end{aligned}
$$

This is the global balance equations for states $P(n, y)$, which thus are fulfilled.

We have thus shown that (15) is a solution to the detailed state probabilities, and as there only exists one solution this is the unique solution. This concludes the proof.

\section{DISTRIBUTION FUNCTION AND MOMENTS OF INDIVIDUAL OVERFLOW TRAFFIC}

For a given number of busy channels on the overflow group, we thus know that the number of connections of each service is Multinomial-distributed. Newcomer \& al. [7] derive the moments of the compound Multinomial distribution. In the following we consider $N=4$ traffic streams. Indexing these as

$$
\{i, j, k, l\}, \quad i \neq j \neq k \neq l
$$

we can derive the following formulas from their results:

$$
\begin{aligned}
E\left(Y_{i}\right) & =p_{i} \varphi_{1} \\
& =p_{i} \beta_{1} \\
& =p_{i} m_{1}
\end{aligned}
$$

$$
\begin{aligned}
E\left(Y_{i} Y_{j}\right) & =p_{i} p_{j} \varphi_{2} \\
& =2 p_{i} p_{j} \beta_{2} \\
& =p_{i} p_{j} m_{2}-p_{i} p_{j} m_{1}
\end{aligned}
$$

$$
\begin{aligned}
E\left(Y_{i}^{2}\right) & =p_{i}^{2} \varphi_{2}+p_{i} \varphi_{1} \\
& =2 p_{i}^{2} \beta_{2}+p_{i} \beta_{1} \\
& =p_{i}^{2} m_{2}+p_{i}\left(1-p_{i}\right) m_{1}
\end{aligned}
$$

$$
\begin{aligned}
E\left(Y_{i} Y_{j} Y_{k}\right) & =p_{i} p_{j} p_{k} \varphi_{3} \\
& =6 p_{i} p_{j} p_{k} \beta_{3} \\
& =p_{i} p_{j} p_{k}\left(m_{3}-3 m_{2}+2 m_{1}\right)
\end{aligned}
$$

$$
\begin{aligned}
E\left(Y_{i}^{2} Y_{j}\right)= & p_{i}^{2} p_{j} \varphi_{3}+p_{i} p_{j} \varphi_{2} \\
= & 6 p_{i}^{2} p_{j} \beta_{3}+2 p_{i} p_{j} \beta_{2} \\
= & p_{i}^{2} p_{j} m_{3}-\left(3 p_{i}^{2} p_{j}-p_{i} p_{j}\right) m_{2} \\
& +\left(2 p_{i}^{2} p_{j}-p_{i} p_{j}\right) m_{1} \\
E\left(Y_{i}^{3}\right)= & p_{i}^{3} \varphi_{3}+3 p_{i}^{2} \varphi_{2}+p_{i} \varphi_{1} \\
= & 6 p_{i}^{3} \beta_{3}+6 p_{i}^{2} \beta_{2}+p_{i} \beta_{1} \\
= & p_{i}^{3} m_{3}+3 p_{i}^{2}\left(1-p_{i}\right) m_{2} \\
& +m_{i}\left(2 p_{i}^{2}-3 p_{i}+1\right) m_{1}
\end{aligned}
$$




$$
\begin{aligned}
E\left(Y_{i} Y_{j} Y_{k} Y_{l}\right)= & p_{i} p_{j} p_{k} p_{l} \varphi_{4} \\
= & 24 p_{i} p_{j} p_{k} p_{l} \beta_{4} \\
= & p_{i} p_{j} p_{k} p_{l}\left(m_{4}-6 m_{3}\right. \\
& \left.+11 m_{2}-6 m_{1}\right) \\
E\left(Y_{i}^{2} Y_{j} Y_{k}\right)= & p_{i}^{2} p_{j} p_{k} \varphi_{4}+p_{i} p_{j} p_{k} \varphi_{3} \\
= & 24 p_{i}^{2} p_{j} p_{k} \beta_{4}+6 p_{i} p_{j} p_{k} \beta_{3} \\
= & p_{i}^{2} p_{j} p_{k} m_{4}+\left(1-p_{i}\right) p_{i} p_{j} p_{k} m_{3} \\
& \quad+\left(11 p_{i}-3\right) p_{i} p_{j} p_{k} m_{2} \\
& \quad-\left(6 p_{1}-2\right) p_{i} p_{j} p_{k} m_{1} \\
E\left(Y_{i}^{2} Y_{j}^{2}\right)= & p_{i}^{2} p_{j}^{2} \varphi_{4}+\left(p_{i}^{2} p_{j}+p_{i} p_{j}^{2}\right) \varphi_{3}+p_{i} p_{j} \varphi_{2} \\
= & 24 p_{i}^{2} p_{j}^{2} \beta_{4}+6\left(p_{i}^{2} p_{j}+p_{i} p_{j}^{2}\right) \beta_{3}+2 p_{i} p_{j} \beta_{2} \\
= & p_{i}^{2} p_{j}^{2} m_{4}-p_{i} p_{j}\left(6 p_{i} p_{j}-p_{i}-p_{j}\right) m_{3} \\
+ & p_{i} p_{j}\left(11 p_{i} p_{j}-3 p_{i}-3 p_{j}+1\right) m_{2} \\
- & p_{i} p_{j}\left(6 p_{i} p_{j}-2 p_{i}-2 p_{j}+1\right) m_{1} \\
= & p_{i}^{3} p_{j} \varphi_{4}+3 p_{i}^{2} p_{j} \varphi_{3}+p_{i} p_{j} \varphi_{2} \\
= & 24 p_{i}^{3} p_{j} \beta_{4}+18 p_{i}^{2} p_{j} \beta_{3}+2 p_{i} p_{j} \beta_{2} \\
= & p_{i}^{3} p_{j} m_{4}-3 p_{i}^{2} p_{j}\left(2 p_{i}-1\right) m_{3} \\
& +p_{i} p_{j}\left(11 p_{i}^{2}-9 p_{i}+1\right) m_{2} \\
& -p_{i} p_{j}\left(6 p_{i}^{2}-1\right) m_{1} \\
E\left(Y_{i}^{3} Y_{j}\right) & \\
& +p_{i}^{4}\left(6 p_{i}^{3}-12 p_{i}^{2}+7 p_{i}-1\right) m_{1} \\
E\left(Y_{i}^{4}\right)= & p_{i}^{4} \beta_{4}+36 p_{i}^{3} \beta_{3}+14 p_{i}^{2} \beta_{2}+p_{i} \beta_{1} \\
= & p_{i} m_{4}-6 p_{i}^{3}\left(p_{i}-1\right) m_{3} \\
& +31)
\end{aligned}
$$

From these formulæ we obtain moments, covariances and correlations coefficients of order up to four dimensions for any combination og traffic streams. For example, we have for two streams $i$ and $j$ :

$$
\operatorname{Cov}\left(Y_{i} Y_{j}\right)=E\left(Y_{i} Y_{j}\right)-E\left(Y_{i}\right) E\left(Y_{j}\right)
$$

\section{IMPLEMENTATION, CONCLUSIONS}

The formulas have been implemented in [3]. The numerical values are stable. We may thus evaluate hierarchical cellular systems where some services are allowed to overflow to overlay cells, whereas other services are blocked or routed elsewhere, when the micro-cell is busy. The overflow systems are dimensioned using well-known methods, for example Equivalent Random Traffic method or Interrupted Poisson Process.

\section{REFERENCES}

[1] Bakmaz, M. (1996): Serving system with the correlated component of overflow traffic having changed serving intensity. IEE Proceedings Communications, Vol. 143 (1996), No. 1, pp. 1-4.

[2] Bakmaz, M.: Analysis of a model of two overflow traffic components with different serving intensisites. AEÜ, International Journal of Electronics and Communications, Vol. 60 (2006), 65-70.
[3] Bækbo, Chr.: Traffic engineering of wireless hierarchichal networks. Master's thesis, Dpt. of Photonics Engineering, Technical University of Denmark 2011. (Supervisor V. B. Iversen). $6+48$ pp.

[4] Descloux, A. (1962): On the components of overflow traffic. Internal Memorandum. Bell Telephone Laboratories Inc., December 1962. 6 pp.

[5] Lindkvist, P.-O.: The Interrupted Poisson Process applied to the generalized Kosten system. International Seminar on Teletraffic and Networks. ISTN-1, Beijing 1987. 37 pp.

[6] Neal, Scotty (1971): Combining correlated streams of nonrandom traffic The Bell system Technical Journal, Vol. 50 (1971): 6, pp. 2015-2037.

[7] Newcomer, J.T. \& Neerchal, N.K. \& Morel, J.G. (2008): Computation of higher order moments from two multinomial overdispersion likelihood models. Department of Mathematics and Statistics, University of Maryland, Baltimore, U.S.A., 2008. 11 pp.

[8] Schehrer, Rudolf G. (1997): A two moments method for overflow systems with different mean holding times. ITC 15, Fifthteenth International Teletraffic congress, Washington 1997. Elsevier Science. Proceedings pp. $1303-1314$.

[9] Schneps-Schneppe, M. \& Sedols, J. (2010): On Erlang-B formula and ERT method extensions. ICUMT-2010, Moscow, October 2010. 6 pp.

[10] Wallström, Bengt (1973): Loss calculations in certain overflow systems where the holding times in successive groups have different means. Ericsson Technics, No. 4, 1973, pp. 177-192.

[11] Zhao, Yongjian \& Gambe, Eiichi (1987): Analysis of partial overflow queueing systems with two kinds of calls. IEEE Transactions on communications, Vol. CPM-35 (1987), No. 9, pp. 942-949. 